\title{
Article \\ Effective Hamiltonians in Nonrelativistic Quantum Electrodynamics
}

\author{
Roberto Passante ${ }^{1,2, *(D)}$ and Lucia Rizzuto ${ }^{1,2}$ (D) \\ 1 Dipartimento di Fisica e Chimica-Emilio Segrè, Università Degli Studi di Palermo, Via Archirafi 36, \\ I-90123 Palermo, Italy; lucia.rizzuto@unipa.it \\ 2 INFN, Laboratori Nazionali del Sud, I-95123 Catania, Italy \\ * Correspondence: roberto.passante@unipa.it
}

Citation: Passante, R.; Rizzuto, L.

Effective Hamiltonians in

Nonrelativistic Quantum

Electrodynamics. Symmetry 2021, 13,

2375. https://doi.org/10.3390/

sym 13122375

Academic Editors: Kayn Forbes and David S. Bradshaw

Received: 14 November 2021

Accepted: 6 December 2021

Published: 9 December 2021

Publisher's Note: MDPI stays neutral with regard to jurisdictional claims in published maps and institutional affiliations.

Copyright: (c) 2021 by the authors. Licensee MDPI, Basel, Switzerland. This article is an open access article distributed under the terms and conditions of the Creative Commons Attribution (CC BY) license (https:/ / creativecommons.org/licenses/by/ $4.0 /)$.

\begin{abstract}
In this paper, we consider some second-order effective Hamiltonians describing the interaction of the quantum electromagnetic field with atoms or molecules in the nonrelativistic limit. Our procedure is valid only for off-energy-shell processes, specifically virtual processes such as those relevant for ground-state energy shifts and dispersion van der Waals and Casimir-Polder interactions, while on-energy-shell processes are excluded. These effective Hamiltonians allow for a considerable simplification of the calculation of radiative energy shifts, dispersion, and Casimir-Polder interactions, including in the presence of boundary conditions. They can also provide clear physical insights into the processes involved. We clarify that the form of the effective Hamiltonian depends on the field states considered, and consequently different expressions can be obtained, each of them with a well-defined range of validity and possible applications. We also apply our results to some specific cases, mainly the Lamb shift, the Casimir-Polder atom-surface interaction, and the dispersion interactions between atoms, molecules, or, in general, polarizable bodies.
\end{abstract}

Keywords: effective Hamiltonian; quantum electrodynamics; dispersion interactions; Casimir-Polder interactions

\section{Introduction}

In molecular quantum electrodynamics, that is, the quantum theory of atoms and molecules interacting with the electromagnetic field in the nonrelativistic limit, several processes of great interest are of a high order in atom-field coupling [1-3]. For example, interatomic dispersion interactions such as van der Waals and Casimir-Polder interactions between two atoms or molecules are fourth-order processes [4-6], and, in the case of three or more atoms, many-body effects start from the sixth order $[1,7,8]$. Additionally, the resonance energy transfer between molecules may involve high-order perturbative calculations in the atom-field coupling [9]. In such cases, the number of relevant Feynman diagrams rapidly grows with the perturbative order, with consequent increasing complexity of the calculations. For this reason, the possibility of finding approximated effective Hamiltonians allowing for the simplification of calculations is very important, and, hopefully, this will also yield a transparent interpretation and physical insights into the relevant physical processes involved [6,10-14]. Effective Hamiltonians can be also used in dynamical (time-dependent) nonequilibrium situations [15]. All these possibilities have fostered the investigation of effective Hamiltonians containing an interaction term that is at least quadratic in the atom-field coupling, where the response of the atom is included in quantities such as, for example, its polarizability, thus allowing for a considerable reduction of the perturbative order required for calculating specific processes and of the number of relevant Feynman diagrams [6]. Very recently, resummation techniques, in which the polarizability is summed to any order, have been developed, and they could also be of great importance for the evaluation of radiative processes such as the Lamb shift and van der Waals interactions for nanostructured materials [16-18]. 
In this paper, we obtain and review different forms of effective Hamiltonians, at the second order in the atom-field coupling, used in nonrelativistic quantum electrodynamics, stressing the range and limit of validity of each of them, as well as their physical interpretation. Application to retarded and nonretarded dispersion interactions and to the atom-surface Casimir-Polder interaction is also discussed. A physical interpretation in terms of the response of the atoms or molecules to vacuum field fluctuations or to real photons is also outlined, as well as the possible role of dissipation in the response function. More specifically, we here present in a clear and organic way the different forms of second-order effective Hamiltonians that can be obtained, depending on the specific field states considered. We also show and stress that the response of the atom is different in the case of vacuum fluctuations or when photons are present; in fact, we find that in the first case, the atom responds through a real function of the frequency, while in the second case through its dynamical polarizability, that has poles in the real frequency axis.

This paper is organized as follows. In Section 2, the general expression of the effective Hamiltonian and of its matrix elements between atom+field states is obtained. In Section 3, specific forms of the effective Hamiltonian in relevant cases are given, and their limits of validity are stressed, as well as the response function of the atoms in the various cases considered, and the possible role of dissipation. Application to interatomic dispersion interactions and to the Casimir-Polder atom-surface interaction, both in the nonretarded and retarded cases, is outlined. Finally, Section 4 is devoted to our conclusive remarks.

\section{The General Expression of the Effective Hamiltonian in Molecular Quantum Electrodynamics}

We first start from the Hamiltonian of one atom, placed at $\mathbf{R}$, interacting with the quantum electromagnetic field, in the multipolar coupling scheme and within dipole approximation [5]. Extension to the case of two or more atoms is straightforward, at the order considered. The Hamiltonian of the system is

$$
\begin{aligned}
H & =H_{0}+H_{I}, \\
H_{0} & =\sum_{\mathbf{k} \lambda} \hbar \omega_{k} a_{\mathbf{k} \lambda}^{\dagger} a_{\mathbf{k} \lambda}+\sum_{\ell} E_{\ell}\left|\phi_{\ell}\right\rangle\left\langle\phi_{\ell}\right|, \\
H_{I} & =-\boldsymbol{\mu} \cdot \mathbf{E}(\mathbf{R})=-\sum_{\ell m} e \mathbf{q}^{\ell m} \cdot \mathbf{E}(\mathbf{R})\left|\phi_{\ell}\right\rangle\left\langle\phi_{m}\right|,
\end{aligned}
$$

where states $\left|\phi_{\ell}\right\rangle$ are a complete set of atomic states with energies $E_{\ell}$, and $a_{\mathbf{k} \lambda}^{\dagger}$ and $a_{\mathbf{k} \lambda}$ are, respectively, creation and annihilation operators relative to the field mode $(\mathbf{k} \lambda)$, with $\lambda=1,2$ being the polarization index and satisfying the usual bosonic commutation relations, and $\omega_{k}=c k ; \mu=e \mathbf{q}$ is the electric dipole moment operator of the atom, with q being the electron coordinate. For simplicity, we assume only one atomic electron is taking part in the radiative process considered, and $\mathbf{q}^{\ell m}=\left\langle\phi_{\ell}|\mathbf{q}| \phi_{m}\right\rangle$ is its matrix element between atomic states. Although this simplification, which is strictly valid for hydrogenlike systems, is not essential in the present calculation, it allows one to include one-electron dipole moment matrix elements only and simplify the discussion of the results; it can, however, be simply overtaken by introducing a full dipole moment operator.

Finally, $\mathbf{E}(\mathbf{r})$ is the electric field operator

$$
\mathbf{E}(\mathbf{r})=\sum_{\mathbf{k} \lambda} \mathbf{E}(\mathbf{k} \lambda ; \mathbf{r})=\sum_{\mathbf{k} \lambda}\left(\mathbf{f}_{\mathbf{k} \lambda}(\mathbf{r}) a_{\mathbf{k} \lambda}+\mathbf{f}_{\mathbf{k} \lambda}^{*}(\mathbf{r}) a_{\mathbf{k} \lambda}^{\dagger}\right),
$$

where $\mathbf{E}(\mathbf{k} \lambda ; \mathbf{r})$ is a Fourier component of the electric field, with $\mathbf{f}_{\mathbf{k} \lambda}(\mathbf{r})$ the field mode functions taking into account the boundary conditions present. In the free space, we have

$$
\mathbf{f}_{\mathbf{k} \lambda}(r)=i \sqrt{\frac{2 \pi \hbar \omega_{k}}{V}} \hat{\mathbf{e}}_{\mathbf{k} \lambda} e^{i \mathbf{k} \cdot \mathbf{r}},
$$


where $\hat{\mathbf{e}}_{\mathbf{k} \lambda}(\lambda=1,2)$ are polarization unit vectors, such that $\hat{\mathbf{e}}_{\mathbf{k} \lambda} \cdot \hat{\mathbf{e}}_{\mathbf{k} \lambda^{\prime}}=\delta_{\lambda \lambda^{\prime}}, \hat{\mathbf{e}}_{\mathbf{k} \lambda} \cdot \hat{\mathbf{k}}=0$, and $V$ is the quantization volume.

The multipolar-coupling Hamiltonian (1) contains also a term equal to $2 \pi \int d^{3} r\left(\mathbf{P}_{\perp}(\mathbf{r})\right)^{2}$, where $\mathbf{P}_{\perp}(\mathbf{r})$ is the transverse part of the polarization field $\mathbf{P}(\mathbf{r})=\sum_{i} e \mathbf{r} \delta\left(\mathbf{r}-\mathbf{r}_{i}\right)$, with $\mathbf{r}_{i}$ being the position of an atomic electron $[1,19,20]$. This is a second-order term in the electric charge that, although it is important for the Lamb shift, does not contribute to the dispersion interactions $[1,6,20,21]$, and for this reason we do not include it, except whenever necessary.

We now follow and briefly review the standard general procedure used in nonrelativistic quantum electrodynamics to obtain a second-order effective Hamiltonian, which consists of applying the following transformation to the Hamiltonian (1)

$$
T=\exp (i e z / \hbar),
$$

where $z$ is an Hermitian operator (with some limitations on the state space where it is defined, as specified later on), chosen in such a way to eliminate, in the transformed Hamiltonian, the first-order terms in the electron charge $e[10,13,14]$. Up to the second order in $e$, we have

$$
\begin{aligned}
T^{-1} H T= & \exp (-i e z / \hbar) H \exp (i e z / \hbar)=H_{0}-e \mathbf{q} \cdot \mathbf{E}(\mathbf{R})+\left[-i e z / \hbar, H_{0}\right] \\
& +[-i e z / \hbar,-e \mathbf{q} \cdot \mathbf{E}(\mathbf{R})]+\frac{1}{2}\left[-i e z / \hbar,\left[-i e z / \hbar, H_{0}\right]\right]+O\left(e^{3}\right) .
\end{aligned}
$$

The operator $z$ is chosen in such a way to make vanishing the first-order terms in the transformed Hamiltonian (5), that is

$$
-e \mathbf{q} \cdot \mathbf{E}(\mathbf{R})-\frac{i e}{\hbar}\left[z, H_{0}\right]=0,
$$

so that the transformed Hamiltonian becomes

$$
\tilde{H}=T^{-1} H T=H_{0}+\frac{i e^{2}}{2 \hbar}[z, \mathbf{q} \cdot \mathbf{E}(\mathbf{R})]=H_{0}+\tilde{H}_{e f f},
$$

with the effective Hamiltonian, expressed in terms of the Hermitian operator $z$, given by

$$
\tilde{H}_{e f f}=\frac{i e^{2}}{2 \hbar}[z, \mathbf{q} \cdot \mathbf{E}(\mathbf{R})] .
$$

This Hamiltonian is at the second order in the atom-field coupling. A second-order energy shift of the system due to the atom-field coupling is then given by the average value of $\tilde{H}_{\text {eff }}$ on the state of the system at hand. Fourth-order corrections, as in the evaluation of interatomic dispersion interactions, can be obtained by a second-order approach if the effective Hamiltonian is used, as explicitly discussed in the next section.

Equation (6) is an implicit relation defining the operator $z$ of the transformation. From this relation, we can obtain its matrix elements between atom+field states of the generic form $\left|\{p\}, \phi_{P}\right\rangle,\{p\}$, and $\phi_{P}$, denoting, respectively, generic field and atom states $(\{p\}$ denotes a general set of number states of the field for all the modes allowed by the boundary conditions), with energy $E_{p}=\sum_{i} \hbar \omega_{i} p_{i}$ and $E_{P}$, respectively. We obtain

$$
\begin{aligned}
& \left\langle\{m\}, \phi_{M}|\mathbf{q} \cdot \mathbf{E}(\mathbf{R})|\{n\}, \phi_{N}\right\rangle \\
& =-\frac{i}{\hbar}\left\langle\{m\}, \phi_{M}|z|\{n\}, \phi_{N}\right\rangle\left(E_{n}-E_{m}+E_{N}-E_{M}\right)
\end{aligned}
$$

Assuming $E_{n}-E_{m}+E_{N}-E_{M} \neq 0$, that is, that the matrix element is taken between unperturbed states with a different energy, we get the matrix elements of the operator $z$

$$
\left\langle\{m\}, \phi_{M}|z|\{n\}, \phi_{N}\right\rangle=i \hbar \frac{\left\langle\{m\}, \phi_{M}|\mathbf{q} \cdot \mathbf{E}(\mathbf{R})|\{n\}, \phi_{N}\right\rangle}{\left(E_{n}+E_{N}\right)-\left(E_{m}+E_{M}\right)}
$$


Equation (10) defines all matrix elements of $z$, except those on the energy shell, that are undefined; thus, the transformation (4) with the matrix elements (10) is not defined on the energy shell. Therefore, the effective Hamiltonian so obtained is not valid when energy-conserving processes are relevant, unless some regularization procedure of the vanishing energy denominators, taking into account dissipation, is used [22-24]. This regularization can be done by adding an imaginary part to the energy denominators shifting the poles to the lower complex frequency half-plane, in agreement with the causality requirement; in our case, this can be done only phenomenologically because we are working within a second-order perturbative approach for a closed system. Because we will obtain effective Hamiltonians mainly to calculate atomic energy shifts and (many-body) dispersion interactions for ground-state systems, where only virtual processes are involved, or when resonant processes are suppressed (in a cavity, for example), in general, this is not a strong limitation for our purposes. However, we wish to point out that our results cannot be directly extended to the case of excited atomic states when resonant processes are present.

For the reasons mentioned above, substitution of the matrix elements (10) of $z$ into (5) allows us to obtain only the off-energy-shell matrix elements of the effective Hamiltonian and not its full expression as an operator in the Hilbert space of the system. For this reason, we can obtain different expressions in terms of field operators, according to the type of field states at hand. The matrix elements of the effective Hamiltonian are

$$
\begin{aligned}
& \left\langle\{m\}, \phi_{M}\left|\tilde{H}_{e f f}\right|\{n\}, \phi_{N}\right\rangle \\
& =-\frac{1}{2} e^{2} \sum_{\{\ell\} L}\left\langle\{m\}, \phi_{M}|\mathbf{q} \cdot \mathbf{E}(\mathbf{R})|\{\ell\}, \phi_{L}\right\rangle\left\langle\{\ell\}, \phi_{L}|\mathbf{q} \cdot \mathbf{E}(\mathbf{R})|\{n\}, \phi_{N}\right\rangle \\
& \times\left(\frac{1}{E_{L}-E_{M}+\sum_{s}\left(\ell_{s}-m_{s}\right) \hbar \omega_{s}}-\frac{1}{E_{N}-E_{L}+\sum_{s}\left(n_{s}-\ell_{s}\right) \hbar \omega_{s}}\right),
\end{aligned}
$$

where the index $s$ indicates photonic modes with frequency $\omega_{s}=c k_{s}$, and the sum over $s$ runs over all field modes. Using the mode expansion (2) for the electric field operator, the matrix elements appearing in the RHS of (11) can be cast in the form

$$
\begin{aligned}
& \left\langle\{m\}, \phi_{M}|\mathbf{q} \cdot \mathbf{E}(\mathbf{R})|\{\ell\}, \phi_{L}\right\rangle\left\langle\{\ell\}, \phi_{L}|\mathbf{q} \cdot \mathbf{E}(\mathbf{R})|\{n\}, \phi_{N}\right\rangle \\
= & \sum_{L} \sum_{\mathbf{k} \lambda \mathbf{k}^{\prime} \lambda^{\prime}}\left\langle\{m\}, \phi_{M}\left|\mathbf{q} \cdot\left(\mathbf{f}_{\mathbf{k}^{\prime} \lambda^{\prime}}(\mathbf{R}) a_{\mathbf{k}^{\prime} \lambda^{\prime}}+\mathbf{f}_{\mathbf{k}^{\prime} \lambda^{\prime}}^{*}(\mathbf{R}) a_{\mathbf{k}^{\prime} \lambda^{\prime}}^{+}\right)\right|\{\ell\}, \phi_{L}\right\rangle \\
& \times\left\langle\{l\}, \phi_{L}\left|\mathbf{q} \cdot\left(\mathbf{f}_{\mathbf{k} \lambda}(\mathbf{R}) a_{\mathbf{k} \lambda}+\mathbf{f}_{\mathbf{k} \lambda}^{*}(\mathbf{R}) a_{\mathbf{k} \lambda}^{+}\right)\right|\{n\}, \phi_{N}\right\rangle .
\end{aligned}
$$

From Equation (12), it is easy to see that, in order that the quantity above be nonvanishing, it is necessary that the total number of photons in $\{m\}$ and $\{n\}$ (that is, the photon number summed over all field modes) must be equal or differ by two. There are thus three possibilities: (i) $\{n\}=\{m\}$, that is elements diagonal in the photon space state; (ii) the difference in $\{n\}$ and $\{m\}$ is all in a single mode $(\overline{\mathbf{k}} \bar{\lambda})$, and thus $m_{\overline{\mathbf{k}} \bar{\lambda}}=n_{\overline{\mathbf{k}} \bar{\lambda}} \pm 2$, with all other modes containing the same number of photons; (iii) the difference is by one photon in each of the two modes $(\overline{\mathbf{k}} \bar{\lambda})$ and $(\tilde{\mathbf{k}} \tilde{\lambda})$, with $(\overline{\mathbf{k}} \bar{\lambda})=n_{\overline{\mathbf{k}} \bar{\lambda}} \pm 1$ and $(\tilde{\mathbf{k}} \tilde{\lambda})=n_{\tilde{\mathbf{k}}} \tilde{\lambda} \pm 1$ (upper or lower sign for both modes), while all other modes in $\{m\}$ and $\{n\}$ have the same photon number.

In the next section, we will explicitly find the form of the effective Hamiltonian for some specific cases, which is relevant in molecular quantum electrodynamics, in particular for atom-surface and (many-body) atom-atom interactions for ground-state atoms or molecules. On the basis of the results obtained in this section, we will now find the relative effective Hamiltonians in the various cases, according to the relevant photon states involved, and point out the range and limit of application of the specific forms obtained. 


\section{Diagonal and Off-Diagonal Matrix Elements of the Effective Hamiltonian}

We now evaluate the matrix elements of the effective Hamiltonian introduced in the previous section. As mentioned in the previous section, the expression of the effective Hamiltonian operator that we obtain, which acts only in the field space, and of its matrix elements, can differ according to the subspace of photon states considered. We work in the Schrödinger representation, and the energy shifts are to be evaluated by time-independent perturbation theory. Related effective Hamiltonians for evaluating energy shifts and dispersion interactions have been also used with a different approach, specifically in the Heisenberg representation in terms of time-dependent field operators, and by separating free and source (scattering) fields [25-29].

We now separately consider different cases; we obtain the relative explicit expressions of the effective Hamiltonian operator (off the energy shell), and apply them to some relevant physical systems, mainly van der Waals and Casimir-Polder dispersion interactions. The possible role of dissipation will be also discussed. The results we obtain are relative to a microscopic description of the system; however, we argue that some aspects of our results could be highly relevant also for macroscopic polarizable bodies and Casimir interactions between macroscopic dielectric bodies in the vacuum.

\subsection{Diagonal Elements of the Effective Hamiltonian and Ground-State Systems (Zero Photons)}

Let us first consider the unperturbed ground state of the system, that is, $\left|\left\{0_{\mathbf{k} \lambda}\right\}, g\right\rangle$, where $\left|\left\{0_{\mathbf{k} \lambda}\right\}\right\rangle$ denotes the photon vacuum state and $|g\rangle$ is the atom's ground state. Due to the atom-field coupling, this state is not an eigenstate of the interacting Hamiltonian, and, as it is well known, the interaction leads to a second-order energy shift that, after mass renormalization, yields the nonrelativistic Lamb shift of the ground-state atom $[5,19,30,31]$. This energy shift is a second-order effect in the electron charge.

Using our effective Hamiltonian (11), this energy shift can be obtained just through a simple first-order calculation, namely, as the expectation value of the effective Hamiltonian on the unperturbed state [14]. In fact, from (11), and using (2), we immediately obtain (Einstein's convention of summation over the repeated indices $i, j$ is assumed)

$$
\begin{aligned}
& \left\langle\left\{0_{\mathbf{k} \lambda}\right\}, g\left|\tilde{H}_{e f f}\right|\left\{0_{\mathbf{k} \lambda}\right\}, g\right\rangle \\
& =-\frac{1}{2} e^{2} \sum_{\mathbf{k} \lambda} \sum_{L} \frac{2\left\langle\left\{0_{\mathbf{k} \lambda}\right\}, g|\mathbf{q} \cdot \mathbf{E}(\mathbf{R})| 1_{\mathbf{k} \lambda}, \phi_{L}\right\rangle\left\langle 1_{\mathbf{k} \lambda}, \phi_{L}|\mathbf{q} \cdot \mathbf{E}(\mathbf{R})|\left\{0_{\mathbf{k} \lambda}\right\}, g\right\rangle}{E_{L g}+\hbar \omega_{k}} \\
& =-\frac{1}{2} \sum_{\mathbf{k} \lambda}\left(\sum_{L} \frac{2 \mu_{i}^{g L} \mu_{j}^{L g}}{E_{L g}+\hbar \omega_{k}}\right)\left\langle\left\{0_{\mathbf{k} \lambda}\right\}\left|E_{i}(\mathbf{k} \lambda ; \mathbf{R}) E_{j}(\mathbf{k} \lambda ; \mathbf{R})\right|\left\{0_{\mathbf{k} \lambda}\right\}\right\rangle,
\end{aligned}
$$

where $\left|1_{\mathbf{k}}\right\rangle$ are one-photon states, $\left|\phi_{L}\right\rangle$ are intermediate atomic states, and $E_{L g}=E_{L}-E_{g}$; also, $E_{L g}>0$, and therefore the quantity in the round brackets in (13) has no poles. It involves off-energy-shell processes only. Thus, in (13) there is no need to introduce dissipation, and, very importantly, the real quantity

$$
\beta_{i j}^{g}\left(\omega_{k}\right)=\sum_{L} \frac{2 \mu_{i}^{g L} \mu_{j}^{L g}}{E_{L g}+\hbar \omega_{k}}
$$

appears, not the atomic dynamic polarizability (see also the following discussion). All relevant properties of the atom are embedded in this function. Equation (13) is the average value on the vacuum state of the electromagnetic field $\left\{0_{\mathbf{k} \lambda}\right\}$ of the following field operator

$$
H_{e f f}^{0}=-\frac{1}{2} \sum_{\mathbf{k} \lambda} \beta_{i j}^{g}\left(\omega_{k}\right) E_{i}(\mathbf{k} \lambda ; \mathbf{R}) E_{j}(\mathbf{k} \lambda ; \mathbf{R}),
$$

that we can take as our effective Hamiltonian within the zero-photon subspace, as specified by the apex 0 . 
By taking the average value of (15) on the field vacuum state $\left|\left\{0_{\mathbf{k} \lambda}\right\}\right\rangle$, we immediately obtain the second-order energy shift due to the atom-field interaction

$$
\Delta E_{g}=\left\langle\left\{0_{\mathbf{k} \lambda}\right\}\left|H_{e f f}^{0}\right|\left\{0_{\mathbf{k} \lambda}\right\}\right\rangle=-\frac{1}{2} \sum_{\mathbf{k} \lambda} \beta_{i j}^{g}\left(\omega_{k}\right)\left[\mathbf{f}_{\mathbf{k} \lambda}^{*}(\mathbf{R})\right]_{i}\left[\mathbf{f}_{\mathbf{k} \lambda}(\mathbf{R})\right]_{j},
$$

where $\mathbf{f}_{\mathbf{k} \lambda}(\mathbf{R})$ are the appropriate mode functions for the system at hand, as introduced in Equation (2), evaluated at the position $\mathbf{R}$ of the atom. For example, in the case of an atom in the unbounded space, Equation (16) yields its ground-state Lamb shift [14] (as previously mentioned, the second-order term proportional to $\mathbf{P}_{\perp}^{2}(\mathbf{r})$ should be added in this case), while for an atom in front of a reflecting plate it yields the atom-surface Casimir-Polder interaction energy at zero temperature [32].

The effective Hamiltonian (15) has a clear physical interpretation. Vacuum electric field fluctuations with wavevector $\mathbf{k}$ induce a dipole moment on the atom given by

$$
\left(\mu_{\text {ind }}^{0}\right)_{i}(\mathbf{k}) \sim \beta_{i j}^{g}\left(\omega_{k}\right) E_{j}(\mathbf{k} \lambda ; \mathbf{R}),
$$

and, in turn, this fluctuating dipole moment interacts with the vacuum field fluctuations yielding (we assume that a vacuum expectation value is taken)

$$
\Delta E \sim-\frac{1}{2} \sum_{\mathbf{k} \lambda}\left(\mu_{\text {ind }}^{0}\right)_{i}(\mathbf{k}) E_{i}(\mathbf{k} \lambda ; \mathbf{R}),
$$

finally yielding (15) and (16). In this case, it is as if a Fourier component of the induced dipole moment with a given $\mathbf{k}$ interacts only with the Fourier component of the electric field having the same $\mathbf{k}$. As we will show in the next subsection, this does not occur when off-diagonal elements of the effective Hamiltonian are relevant, as in the case of two- and many-body dispersion interactions between atoms or molecules.

A very important point is that the response of the atom to the vacuum field fluctuations is not through its dynamical polarizability but through the function $\beta_{i j}^{g}\left(\omega_{k}\right)$, as defined by (14), which is a real quantity at any frequency without poles and related dissipative properties. This does not contrast with the fluctuation-dissipation theorem and the Kramers-Krönig dispersion relations, which indeed consider the response of a system to an external applied field (linear response theory), while in our case only vacuum field fluctuations act, which cannot induce real transitions. This result could be important also for macroscopic polarizable bodies and their role as boundary conditions in the Casimir effect, as well as for the long-lasting dispute in the literature about the most appropriate dielectric model (plasma or Drude model, for example) to be used in the Casimir effect for dielectrics [33-35]. We will address this point in a forthcoming publication.

From Equations (11) and (12), we can also obtain diagonal matrix elements of the effective Hamiltonian in the case of one mode populated with $n_{\mathbf{k} \lambda}$ photons, with all other field modes being in their vacuum state, obtaining

$$
\begin{aligned}
& \left\langle n_{\mathbf{k} \lambda}, \phi_{N}\left|\tilde{H}_{e f f}\right| n_{\mathbf{k} \lambda}, \phi_{N}\right\rangle \\
& =-\frac{1}{2} \sum_{L}\left|\boldsymbol{\mu}^{N L} \cdot \mathbf{E}(\mathbf{k} \lambda ; \mathbf{R})\right|^{2}\left(2 n_{\mathbf{k} \lambda} \frac{2 E_{L N}}{E_{L N}^{2}-\left(\hbar \omega_{k}\right)^{2}}+\frac{2}{E_{L N}+\hbar \omega_{k}}\right) \\
& =-\frac{1}{2} E_{i}(\mathbf{k} \lambda ; \mathbf{R}) E_{j}(\mathbf{k} \lambda ; \mathbf{R})\left(2 n_{\mathbf{k} \lambda} \alpha_{i j}\left(\omega_{k}\right)+\beta_{i j}\left(\omega_{k}\right)\right),
\end{aligned}
$$

where

$$
\alpha_{i j}\left(\omega_{k}\right)=\sum_{L} \frac{2 E_{L N} \mu_{i}^{N L} \mu_{j}^{L N}}{E_{L N}^{2}-\left(\hbar \omega_{k}\right)^{2}}
$$


is the ground-state atomic dynamic polarizability, with poles at $\hbar \omega_{k}=E_{L N}$ and obeying the dispersion relations, and

$$
\beta_{i j}^{N}\left(\omega_{k}\right)=\sum_{L} \frac{2 \mu_{i}^{N L} \mu_{j}^{L N}}{E_{L N}+\hbar \omega_{k}}
$$

is a function analogous to (14) for the generic atomic state $N$. Equation (21) has no poles if $N$ is the ground state, as already discussed, while it can have poles in the case of other atomic states. We wish to point out and stress that Equation (19) clearly shows that the atom responds to real photons through its dynamical polarizability $\alpha_{i j}\left(\omega_{k}\right)$ and to the vacuum fluctuating field through the $\beta_{i j}^{N}\left(\omega_{k}\right)$ function.

For atoms or molecules with a random orientation, being $\left\langle\mu_{i}^{g L} \mu_{j}^{L g}\right\rangle=\left|\boldsymbol{\mu}^{g L}\right|^{2} \delta_{i j} / 3$, Equation (15) becomes

$$
H_{e f f}^{(0)}=-\frac{1}{2} \sum_{\mathbf{k} \lambda} \beta_{a v}^{g}\left(\omega_{k}\right)(E(\mathbf{k} \lambda ; \mathbf{R}))^{2},
$$

with

$$
\beta_{a v}^{g}\left(\omega_{k}\right)=\frac{2}{3} \sum_{L} \frac{\left|\mu^{L g}\right|^{2}}{E_{L g}+\hbar \omega_{k}} .
$$

If the relevant frequencies of the field are such that $\omega_{k} \ll E_{L g} / \hbar$, then $\beta_{a v}^{g}\left(\omega_{k}\right) \simeq$ $\beta_{a v}^{g}(0)=\alpha^{g}$, where $\alpha^{g}$ is the isotropic static polarizability of the ground-state atom. Then, Equation (22) reduces to $H_{e f f}^{(0)} \simeq-\frac{1}{2} \alpha E^{2}(\mathbf{R})$; we wish to stress that only in this limiting case, $\omega_{k} \rightarrow 0$, our $\beta$ function coincides with the (static) polarizability of the atom. This form of the effective Hamiltonian has been used, for example, for evaluating the far-zone (retarded) dispersion interaction of an atom placed near a conducting wall [27].

\subsection{Off-Diagonal Elements of the Effective Hamiltonian}

We now consider the off-diagonal elements of the effective Hamiltonian between atom-field states, which we can obtain from (11) and (12); as mentioned at the end of the previous section, in this case the total number of photons in the two states must differ by two. We find

$$
\begin{aligned}
& \left\langle\{p\}, n_{\mathbf{k} \lambda}+1, m_{\mathbf{k}^{\prime} \lambda^{\prime}}+1, \phi_{M}\left|\tilde{H}_{e f f}\right|\{p\}, n_{\mathbf{k} \lambda}, m_{\mathbf{k}^{\prime} \lambda^{\prime}}, \phi_{N}\right\rangle \\
= & -\frac{1}{2} e^{2} \sum_{L}\left\langle\phi_{M}\left|q_{i}\right| \phi_{L}\right\rangle\left\langle\phi_{L}\left|q_{j}\right| \phi_{N}\right\rangle \sqrt{n_{\mathbf{k} \lambda}+1} \sqrt{m_{\mathbf{k}^{\prime} \lambda^{\prime}}+1}\left(\mathbf{f}_{\mathbf{k} \lambda}^{*}(\mathbf{R})\right)_{i}\left(\mathbf{f}_{\mathbf{k}^{\prime} \lambda^{\prime}}^{*}(\mathbf{R})\right)_{j} \\
& \times\left(\frac{1}{E_{L M}+\hbar \omega_{k}}-\frac{1}{E_{N L}+\hbar \omega_{k^{\prime}}}\right)+\left(\mathbf{k} \lambda \leftrightarrow \mathbf{k}^{\prime} \lambda^{\prime}\right),
\end{aligned}
$$

where $\{p\}$ indicates all modes different from $\mathbf{k} \lambda$ and $\mathbf{k}^{\prime} \lambda^{\prime}, \mathbf{k} \lambda \neq \mathbf{k}^{\prime} \lambda^{\prime}$.

We can specialize Equation (24) to the case $n_{\mathbf{k} \lambda}=m_{\mathbf{k}^{\prime} \lambda^{\prime}}=0,\{p\}=\{0\}$ (vacuum state for all other field modes), $\phi_{M}=\phi_{N}$, relevant for the calculation of two- or threebody dispersion interactions between atoms or molecules in the vacuum space at zero temperature, allowing one to reduce, respectively, a fourth or sixth-order calculation to a second- or third-order calculation [10],

$$
\begin{aligned}
& \left\langle\{0\}, 1_{\mathbf{k} \lambda}, 1_{\mathbf{k}^{\prime} \lambda^{\prime}}, \phi_{N}\left|\tilde{H}_{e f f}\right|\{0\}, 0_{\mathbf{k} \lambda}, 0_{\mathbf{k}^{\prime} \lambda^{\prime}}, \phi_{N}\right\rangle \\
= & -\frac{1}{2}\left(\mathbf{f}_{\mathbf{k} \lambda}^{*}(\mathbf{R})\right)_{i}\left(\mathbf{f}_{\mathbf{k}^{\prime} \lambda^{\prime}}^{*}(\mathbf{R})\right)_{j} \sum_{L}\left(\frac{2 E_{L N} \mu_{i}^{N L} \mu_{j}^{L N}}{E_{L N}^{2}-\left(\hbar \omega_{k}\right)^{2}}+\frac{2 E_{L N} \mu_{i}^{N L} \mu_{j}^{L N}}{E_{L N}^{2}-\left(\hbar \omega_{k^{\prime}}\right)^{2}}\right) \\
= & -\frac{1}{2}\left(\mathbf{f}_{\mathbf{k} \lambda}^{*}(\mathbf{R})\right)_{i}\left(\mathbf{f}_{\mathbf{k}^{\prime} \lambda^{\prime}}^{*}(\mathbf{R})\right)_{j}\left(\alpha_{i j}\left(\omega_{k}\right)+\alpha_{i j}\left(\omega_{k^{\prime}}\right)\right),
\end{aligned}
$$

where the symmetry between primed and non primed $(\mathbf{k} \lambda)$ s has been exploited, and the atomic matrix elements $\mu^{L N}$ can be taken real without loss of generality. This quantity is the 
matrix element on the field states considered, $\left|\{0\}, 0_{\mathbf{k} \lambda}, 0_{\mathbf{k}^{\prime} \lambda^{\prime}}, \phi_{N}\right\rangle$ and $\left|\{0\}, 1_{\mathbf{k} \lambda}, 1_{\mathbf{k}^{\prime} \lambda^{\prime}}, \phi_{N}\right\rangle$ of the following operator

$$
H_{e f f}^{\prime}=-\frac{1}{2} \sum_{\mathbf{k} \lambda} \alpha_{i j}\left(\omega_{k}\right) E_{i}(\mathbf{k} \lambda ; \mathbf{R}) E_{j}(\mathbf{R}),
$$

that we can take as the effective Hamiltonian in the field subspace spanned by the states considered. The properties of the atoms or molecules relevant for the processes involved are contained in their dynamical polarizability, $\alpha_{i j}\left(\omega_{k}\right)$, and the effective Hamiltonian acts on the photon subspace only.

The effective Hamiltonian (26) is different from (15), and its physical interpretation is different. The response of the atom in the present case is not through the $\beta_{i j}\left(\omega_{k}\right)$ function but through its dynamical polarizability, and the interaction of the $\mathbf{k}$ Fourier component of the induced dipole moment is with the total electric field and not with only its $\mathbf{k}$ component, as in the previous diagonal case (18).

If the frequency of the (virtual) photons involved is much smaller than the relevant atomic transition frequencies, we can approximate the dynamical polarizabiblity with the static one, $\alpha_{i j}\left(\omega_{k}\right) \simeq \alpha_{i j}(0)=\alpha_{i j}$, and (26) reduce it to

$$
H_{e f f}^{s t}=-\frac{1}{2} \sum_{\mathbf{k} \lambda} \alpha_{i j} E_{i}(\mathbf{k} \lambda ; \mathbf{R}) E_{j}(\mathbf{R})=-\frac{1}{2} \alpha_{i j} E_{i}(\mathbf{R}) E_{j}(\mathbf{R}),
$$

which can be used to obtain retarded far-zone dispersion interactions, where only the contribution of low-frequency virtual photons is relevant [13].

A straightworward application of the effective Hamiltonian (26) is the evaluation of dispersion (van der Waals and Casimir-Polder) interactions between two ground-state atoms in the vacuum (zero temperature), even when boundary conditions are present. In this case, we have two atoms, labeled as A and B, respectively, located at $\mathbf{R}_{A}$ and $\mathbf{R}_{b}$. The standard quantum electrodynamical calculation of their dispersion interaction involves a fourth-order perturbative calculation [4,21], while the use of the effective Hamiltonian (26) allows us to reduce it to a much simpler second-order calculation. The Hamiltonian of the system is $H=H_{A}+H_{B}+H_{F}+H_{e f f}^{\prime}(A)+H_{e f f}^{\prime}(B)$, where $H_{A}$ and $H_{B}$ are, respectively, the Hamiltonian of atoms $\mathrm{A}$ and $\mathrm{B} ; H_{F}$ is the free field Hamiltonian; and $H_{e f f}^{\prime}(A)$ and $H_{e f f}^{\prime}(B)$ are the effective Hamiltonian relative to atoms $\mathrm{A}$ and $\mathrm{B}$, respectively. The secondorder energy shift in the polarizabilities, including only terms containing the position of both atoms (the other terms do not contribute to their interaction energy), is

$$
\Delta E_{A B}=\sum_{\mathbf{k} \lambda \mathbf{k}^{\prime} \lambda^{\prime}} \frac{\left\langle\left\{0_{\mathbf{k} \lambda}\right\}\left|H_{e f f}^{\prime}(A)\right| 1_{\mathbf{k} \lambda} 1_{\mathbf{k}^{\prime} \lambda^{\prime}}\right\rangle\left\langle 1_{\mathbf{k} \lambda} 1_{\mathbf{k}^{\prime} \lambda^{\prime}}\left|H_{e f f}^{\prime}(B)\right|\left\{0_{\mathbf{k} \lambda}\right\}\right\rangle}{-\hbar\left(\omega_{k}+\omega_{k^{\prime}}\right)}+(A \leftrightarrow B) .
$$

We wish to point out that, although our effective Hamiltonian is a second-order one in the atomic dipole moments (see Equation (5)), in the present case of two atoms we can take the two dipole moments $\mu_{A}$ and $\mu_{B}$ as independent expansion parameters, and thus (28) is a second-order quantity in $\mu_{A}$ and in $\mu_{B}$. Fourth-order terms in $\mu_{A}$ and in $\mu_{B}$, which would yield the fourth-order correction to the Lamb shift (not depending from the interatomic distance), would require a fourth-order effective Hamiltonian and are here of course neglected, coherently with our approximations.

Taking into account the form (26) of the effective Hamiltonian, and using (2), we get

$$
\begin{aligned}
\Delta E_{A B}= & -\frac{1}{4 \hbar} \sum_{\mathbf{k} \lambda \mathbf{k}^{\prime} \lambda^{\prime}} \frac{\alpha_{A}\left(\omega_{k}\right) \alpha_{B}\left(\omega_{k}\right)}{\omega_{k}+\omega_{k^{\prime}}}\left[\mathbf{f}_{\mathbf{k} \lambda}\left(\mathbf{R}_{A}\right) \cdot \mathbf{f}_{\mathbf{k}^{\prime} \lambda^{\prime}}\left(\mathbf{R}_{A}\right) \mathbf{f}_{\mathbf{k} \lambda}^{*}\left(\mathbf{R}_{B}\right) \cdot \mathbf{f}_{\mathbf{k}^{\prime} \lambda^{\prime}}^{*}\left(\mathbf{R}_{B}\right)\right. \\
& \left.+\mathbf{f}_{\mathbf{k} \lambda}\left(\mathbf{R}_{B}\right) \cdot \mathbf{f}_{\mathbf{k}^{\prime} \lambda^{\prime}}\left(\mathbf{R}_{B}\right) \mathbf{f}_{\mathbf{k} \lambda}^{*}\left(\mathbf{R}_{A}\right) \cdot \mathbf{f}_{\mathbf{k}^{\prime} \lambda^{\prime}}^{*}\left(\mathbf{R}_{A}\right)\right]
\end{aligned}
$$

where $\alpha_{A}\left(\omega_{k}\right)$ and $\alpha_{B}\left(\omega_{k}\right)$ are, respectively, the dynamic polarizability of atoms A and B. 
For atoms in the free space and using the mode functions (3), we obtain

$$
\Delta E_{A B}=-\frac{2 \pi^{2} \hbar}{V^{2}} \sum_{\mathbf{k} \lambda \mathbf{k}^{\prime} \lambda^{\prime}} \frac{\alpha_{A}\left(\omega_{k}\right) \alpha_{B}\left(\omega_{k}\right)}{\omega_{k}+\omega_{k^{\prime}}}\left(\hat{\mathbf{e}}_{\mathbf{k} \lambda} \cdot \hat{\mathbf{e}}_{\mathbf{k}^{\prime} \lambda^{\prime}}\right)^{2} e^{i\left(\mathbf{k}+\mathbf{k}^{\prime}\right) \cdot \mathbf{R}},
$$

where $\mathbf{R}=\mathbf{R}_{A}-\mathbf{R}_{B}$ is the distance between the two atoms or molecules (we have assumed real polarization unit vector). Explicit evaluation of (30), using $\sum_{\lambda}\left(\hat{\mathbf{e}}_{\mathbf{k} \lambda}\right)_{i}\left(\hat{\mathbf{e}}_{\mathbf{k} \lambda}\right)_{j}=\delta_{i j}-\hat{k}_{i} \hat{k}_{j}$ for the polarization sums and in the continuum limit $\sum_{\mathbf{k}} \rightarrow\left(V /(2 \pi)^{3}\right) \int d^{3} k$, yields the well-known dispersion interaction energy between two isotropic atoms as obtained through standard fourth-order perturbation theory, by summing the contributions of the twelve relevant time-ordered Feynman diagrams [4,6,21]. It scales as $R^{-6}$ in the near zone (nonretarded regime: $R \ll c / \omega_{0}, \omega_{0}$ being a main transition angular frequency of the atom) and as $R^{-7}$ in the far zone (retarded Casimir-Polder regime, $R \gg c / \omega_{0}$ ); thus, the (attractive) force between the two atoms scales as $R^{-7}$ and $R^{-8}$ in the near and far zone, respectively. Typical values of the dispersion force between two hydrogen atoms are: $\sim 10^{-30} \mathrm{~N}$ for $R=10^{-7} \mathrm{~m}$ (near zone) and $\sim 10^{-37} \mathrm{~N}$ for $R=10^{-6} \mathrm{~m}$ (far zone). If the appropriate mode functions are used, Equation (29) can be also exploited to evaluate the dispersion interaction in the presence of generic metallic boundaries, for example, a reflecting mirror [36,37]. These results could in principle be extended to generic electrically polarizable bodies. For systems of three or more macroscopic bodies, the non-additivity of dispersion interactions should, however, be taken into account $[27,38]$, and effective Hamiltonians can be very useful in dealing with nonadditive interactions.

\section{Conclusions}

In this paper, we have considered second-order effective Hamiltonians in nonrelativistic quantum electrodynamics, which are very useful to obtain several radiative processes through simpler calculations, compared to the usual evaluations based on the multipolarcoupling Hamiltonian. Examples are energy shifts and Casimir-Polder atom-surface interactions, as well as two- and many-body dispersion interactions between atoms or molecules, in both the nonretarded (near zone) and the retarded (far zone) regime, even in the presence of reflecting boundary conditions. We have reviewed and analyzed different expressions of the effective Hamiltonian, according to the specific system considered and the field states, stressing their limit of validity. The presence or absence of poles in the response function of the atoms has been carefully analyzed, showing that in the case of the photon vacuum the response function has no poles and it is a real function, while in the other cases it is the atomic dynamical polarizability, obeying the Kramers-Krönig dispersion relations. In a forthcoming publication, we will address possible extensions of these results to macroscopic polarizable bodies and Casimir interactions between macroscopic dielectric bodies, stressing in particular their relevance for the dielectric model to be used for the evaluation of such interactions between macroscopic bodies.

Author Contributions: Conceptualization, R.P.; methodology, R.P. and L.R.; formal analysis, R.P. and L.R.; investigation, R.P. and L.R.; writing-original draft preparation, R.P.; writing-review and editing, L.R. All authors have read and agreed to the published version of the manuscript.

Funding: This research received no external funding.

Acknowledgments: The authors gratefully acknowledge financial support from the Julian Schwinger Foundation and MUR.

Conflicts of Interest: The authors declare no conflict of interest. 


\section{References}

1. Salam, A. Molecular Quantum Electrodynamics; McGraw-Hill: Singapore, 2010.

2. Salam, A. Molecular quantum electrodynamics in the Heisenberg picture: A field theoretic viewpoint. Int. Rev. Phys. Chem. 2008, 27, 405-448. [CrossRef]

3. Milonni, P. An Introduction to Quantum Optics and Quantum Fluctuations; Oxford University Press: London, UK, 2019.

4. Casimir, H.B.G.; Polder, D. The Influence of Retardation on the London-van der Waals Forces. Phys. Rev. 1948, 73, 360-372. [CrossRef]

5. Compagno, G.; Passante, R.; Persico, F. Atom-Field Interactions and Dressed Atoms; Cambridge Universty Press: Cambridge, UK, 1995.

6. Passante, R. Dispersion Interactions between Neutral Atoms and the Quantum Electrodynamical Vacuum. Symmetry 2018, 10, 735. [CrossRef]

7. Aub, M.R.; Zienau, S.; Massey, H.S.W. Studies on the retarded interaction between neutral atoms - I. Three-body London-van der Waals interaction of neutral atoms. Proc. R. Soc. Lond. Ser. A Math. Phys. Sci. 1960, 257, 464-476.

8. Aldegunde, J.; Salam, A. Dispersion energy shifts among N bodies with arbitrary electric multipole polarisability: Molecular QED theory. Mol. Phys. 2015, 113, 226-231. [CrossRef]

9. Salam, A. Bridge-Mediated RET between Two Chiral Molecules. Appl. Sci. 2021, 11, 1012. [CrossRef]

10. Passante, R.; Power, E.; Thirunamachandran, T. Radiation-molecule coupling using dynamic polarizabilities: Application to many-body forces. Phys. Lett. A 1998, 249, 77-82. [CrossRef]

11. Barcellona, P.; Safari, H.; Salam, A.; Buhmann, S.Y. Enhanced Chiral Discriminatory van der Waals Interactions Mediated by Chiral Surfaces. Phys. Rev. Lett. 2017, 118, 193401. [CrossRef]

12. Buhmann, S.Y.; Salam, A. Three-Body Dispersion Potentials Involving Electric Octupole Coupling. Symmetry 2018, 10, 343. [CrossRef]

13. Craig, D.P.; Power, E.A. The asymptotic Casimir-Polder potential from second-order perturbation theory and its generalization for anisotropic polarizabilities. Int. J. Quantum Chem. 1969, 3, 903-911. [CrossRef]

14. Passante, R.; Power, E. The Lamb shift in non-relativistic quantum electrodynamics. Phys. Lett. A 1987, 122, 14-16. [CrossRef]

15. Rizzuto, L.; Passante, R.; Persico, F. Dynamical Casimir-Polder energy between an excited- and a ground-state atom. Phys. Rev. A 2004, 70, 012107. [CrossRef]

16. Flick, J.; Schäfer, C.; Ruggenthaler, M.; Appel, H.; Rubio, A. Ab Initio Optimized Effective Potentials for Real Molecules in Optical Cavities: Photon Contributions to the Molecular Ground State. ACS Photonics 2018, 5, 992-1005. [CrossRef] [PubMed]

17. Haugland, T.S.; Schäfer, C.; Ronca, E.; Rubio, A.; Koch, H. Intermolecular interactions in optical cavities: An ab initio QED study. J. Chem. Phys. 2021, 154, 094113. [CrossRef] [PubMed]

18. Schäfer, C.; Buchholz, F.; Penz, M.; Ruggenthaler, M.; Rubio, A. Making ab initio QED functional(s): Nonperturbative and photon-free effective frameworks for strong light-matter coupling. Proc. Natl. Acad. Sci. USA 2021, 118, e2110464118. [CrossRef]

19. Power, E. Introductory Quantum Electrodynamics; Longmans: Oxford, UK, 1964.

20. Milonni, P. Semiclassical and quantum-electrodynamical approaches in nonrelativistic radiation theory. Phys. Rep. 1976, $25,1-81$. [CrossRef]

21. Craig, D.; Thirunamachandran, T. Molecular Quantum Electrodynamics; Courier Corporation: Mineola, NY, USA, 1998.

22. Andrews, D.L.; Naguleswaran, S.; Stedman, G.E. Phenomenological damping of nonlinear-optical response tensors. Phys. Rev. A 1998, 57, 4925-4929. [CrossRef]

23. Milonni, P.W.; Boyd, R.W. Influence of radiative damping on the optical-frequency susceptibility. Phys. Rev. A 2004, 69, 023814. [CrossRef]

24. Milonni, P.W.; Loudon, R.; Berman, P.R.; Barnett, S.M. Linear polarizabilities of two- and three-level atoms. Phys. Rev. A 2008, 77, 043835. [CrossRef]

25. Power, E.A.; Thirunamachandran, T. Quantum electrodynamics with nonrelativistic sources. III. Intermolecular interactions. Phys. Rev. A 1983, 28, 2671-2675. [CrossRef]

26. Shahmoon, E. Van der Waals and Casimir-Polder dispersion forces. In Forces of the Quantum Vacuum: An Introduction to Casimir Physics; Simpson, W., Leonhardt, U., Eds.; World Scientific: Singapore, 2015; pp. 61-106.

27. Milonni, P. The Quantum Vacuum: An Introduction to Quantum Electrodynamics; Academic Press: San Diego, CA, USA, 1994.

28. Milonni, P.W.; Smith, A. van der Waals dispersion forces in electromagnetic fields. Phys. Rev. A 1996, 53, 3484-3489. [CrossRef]

29. Milonni, P.W. Casimir effects. Phys. Scr. 2007, 76, C167-C171. [CrossRef]

30. Bethe, H.A. The Electromagnetic Shift of Energy Levels. Phys. Rev. 1947, 72, 339-341. [CrossRef]

31. Maclay, G.J. History and Some Aspects of the Lamb Shift. Physics 2020, 2, 105-149. [CrossRef]

32. Messina, R.; Passante, R.; Rizzuto, L.; Spagnolo, S.; Vasile, R. Casimir-Polder forces, boundary conditions and fluctuations. J. Phys. A Math. Theor. 2008, 41, 164031. [CrossRef]

33. Bordag, M.; Klimchitskaya, G.; Mohideen, U.; Mostepanenko, V. Advances in the Casimir Effect; Oxford Science Publications: Oxford, UK, 2009.

34. Mostepanenko, V.M. Casimir Puzzle and Casimir Conundrum: Discovery and Search for Resolution. Universe 2021, 7, 84. [CrossRef]

35. Brevik, I.; Shapiro, B. A critical discussion of different methods and models in Casimir effect. arXiv 2021, arXiv:2109.15155. 
36. Power, E.A.; Thirunamachandran, T. Quantum electrodynamics in a cavity. Phys. Rev. A 1982, 25, 2473-2484. [CrossRef]

37. Spagnolo, S.; Passante, R.; Rizzuto, L. Field fluctuations near a conducting plate and Casimir-Polder forces in the presence of boundary conditions. Phys. Rev. A 2006, 73, 062117. [CrossRef]

38. Barcellona, P.; Passante, R. A microscopic approach to Casimir and Casimir-Polder forces between metallic bodies. Ann. Phys. 2015, 355, 282-292. [CrossRef] 